\title{
Electrodeposition of Polypyrrol/Platinum Films
}

\author{
Tiffany A. Marín, Franklin J. Isaza, Jorge A. Calderón \\ Grupo de Corrosión y Protección, Grupo de Materiales Electroactivos, \\ Universidad de Antioquia, Medellín, Colombia
}

Received 25 April 2008; accepted 14 January 2009

\begin{abstract}
The fuel cells are new alternatives for energy production with low environmental impact. Nowadays, conducting polymers are considered the most promising material to manufacture these type of cells because of their easy application and operation, being polypyrrole (PPy) one of them. The electrochemical synthesis of single-layer polypyrrole and two-layers or multiple-layers polypyrrole/platinum ( $\mathrm{PPy} / \mathrm{Pt})$ films is reported in this paper. The films were obtained by electropolymerisation of pyrrole and cathodic deposition of platinum from ammonium hexachloroplatinate salt on stainless steel 304. The composition, morphology, conductivity and the electrochemical properties of the films were study by Raman Spectroscopy, SEM, Profilometer, impedance and cyclic voltammetry. The possible use of PPy and PPy/Pt films as electrodes for fuel cells is also discussed.
\end{abstract}

Keywords: polypyrrole/platinum, conducting polymers, cyclic voltammetry.

\section{Introducción}

Los polímeros conductores son un tipo de materiales que tienen comportamiento redox, además de propiedades combinadas de metales y plásticos. Estos han sido estudiados por sus potenciales aplicaciones como: dispositivos para almacenar carga, materiales para baterías, celdas solares, electrodos modificados, recubrimientos anticorrosivos y transistores moleculares, entre otras [1,2]. Estos polímeros se perfilan como materiales prometedores para la fabricación de celdas de combustible en términos de su aplicación y operación, siendo el polipirrol (PPy) uno de ellos. El PPy presenta alta conductividad eléctrica, estabilidad térmica, resistencia a la oxidación, es de fácil obtención, puede formarse a partir de disoluciones acuosas y en disolventes orgánicos [3]. Además puede obtenerse por polimerización química o electroquímica considerando que la electropolimerización proporciona un mejor control del espesor y de la

\footnotetext{
* Corresponding author. E-mail address: franklinj@udea.edu.co
} 
morfología de las películas [2]. Actualmente, con el fin de disminuir costos en la producción de celdas de combustible, se buscan alternativas con igual o mejor eficiencia a la de los metales empleados en los electrodos y un ejemplo de éstos son los materiales compuestos de matriz polimérica conductora [1,3-5]. Con este fin se ha estudiado la electrodeposición de PPy con incorporación de platino, plata u oro, en experimentos independientes, sobre sustratos tales como: carbono vítreo, oro y platino [1,4-9]. Adicionalmente, se han reportado estudios de electrodeposición de PPy sobre otros sustratos tales como acero inoxidable 304 y 316L, donde se evalúan propiedades morfológicas, propiedades anticorrosivas y en aplicaciones como biosensores [2,10-28]. Los sustratos de acero inoxidable, utilizados como soporte en sistemas poliméricos modificados con partículas catalíticas, son muy atractivos para las aplicaciones tecnológicas. Considerando que es un material económico y además, que el acero inoxidable puede sufrir una modificación interfacial con la formación de una película delgada de óxidos que conducen a una pasivación del mismo [29,30]. En este trabajo se presenta la obtención electroquímica de películas de PPy y PPy/Pt depositadas sobre acero inoxidable 304, las cuales se caracterizaron por medio de espectroscopia Raman, microscopía electrónica de barrido, perfilometría, impedancia y se evaluaron sus potenciales de oxidación por voltametría cíclica. Las películas fueron sintetizadas electroquímicamente en presencia de su monómero pirrol y de la sal de hexacloroplatinato de amonio obtenida por tratamiento hidrometalúrgico.

\section{Procedimiento experimental}

Para la síntesis electroquímica de recubrimientos de polipirrol y polipirrol/platino se usó Pirrol (GR, Merck) sin destilar, $\mathrm{H}_{2} \mathrm{SO}_{4}$ (96\%, Merck), $\mathrm{HCl}$ (37\%, Merck), $\mathrm{HNO}_{3}$ (65\%, Merck), $\mathrm{NH}_{4} \mathrm{Cl}$ (GR, Merck) y Platino aluvial. Todas las disoluciones utilizadas fueron preparadas con agua destilada $\left(5,0 \mu \mathrm{S} \cdot \mathrm{cm}^{-1}\right)$. Para obtener el hexacloroplatinato de amonio $\left[\left(\mathrm{NH}_{4}\right)_{2} \mathrm{PtCl}_{6}\right]$ a partir de platino aluvial, se usaron procedimientos hidrometalúrgicos reportados en la literatura [31,32]. Esta sal compleja se usó como fuente de Pt para la síntesis electroquímica. La obtención de la sal de platino obedece a la necesidad de reducir costos en las materias primas usadas en la síntesis electroquímica, pues localmente se cuenta con platino aluvial de bajo costo. La electropolimerización y todos los ensayos electroquímicos se realizaron en una atmósfera de $\mathrm{N}_{2}$, en una celda de vidrio de tres electrodos: como electrodo de trabajo se usó un disco de acero inoxidable 304, con diámetro de $19 \mathrm{~mm}$. Antes de cada experimento, estos discos se pulieron mecánicamente hasta un grado estándar de 600. Posteriormente se lavaron con agua destilada y se desengrasaron con etanol. El sustrato de acero fue pasivado antes de cada electrodeposición. Para ello, a partir del potencial de circuito abierto se llevó a un potencial de $1,0 \mathrm{~V}$, con una velocidad de barrido de $100 \mathrm{mV} \mathrm{s}^{-1}$. Además se emplearon un electrodo auxiliar de $\mathrm{Pt}$, en forma de rejilla cilíndrica con un diámetro de $4 \mathrm{~cm}$ y una altura de $5 \mathrm{~cm}$ (área $=62,83 \mathrm{~cm}^{2}$ ) y un electrodo de referencia de calomel saturado (ECS). Todos los potenciales indicados en este trabajo están referidos a este electrodo ECS. Para la electrodeposición y caracterización electroquímica, se empleó un potenciostato- 
galvanostato PGST-30 Autolab. La composición estructural de las películas se analizó en un espectrofotómetro Raman RENISHAW RAMAN IMAGINE System 3000, acoplado a un Microscopio Olympus con detector CCD (Wright 600 x 400 pixels), usando un Láser He/Ne (Spectra Physics mod. 127), con una longitud de onda de 632,8 nm (rojo). Las propiedades morfológicas, con un microscopio electrónico de barrido JEOL-5910LV y un JEOL JSM-6490LV. El espesor, usando un perfilómetro Veeco instruments DEKTAK 8 stylus profilometer.

Tabla 1. Descripción de los experimentos,

\begin{tabular}{||l|l||}
\hline \multirow{3}{*}{ Bicapa de PPy/Pt } & $\begin{array}{l}\text { 1. } \begin{array}{l}\text { Obtención de películas de } \\
\text { PPy. } \\
\text { 2. }\end{array} \\
\text { Reducción de Pt (IV) modo: } \\
\text { - Potenciostático: PPy/Pt 1 } \\
\text { - Galvanostático: PPy/Pt } 2\end{array}$ \\
\hline Multicapa de PPy/Pt & $\begin{array}{l}\text { 1. Galvanostático: PPy/Pt 3. } \\
\text { 2. Potenciostático: PPy/Pt 4 }\end{array}$ \\
\hline
\end{tabular}

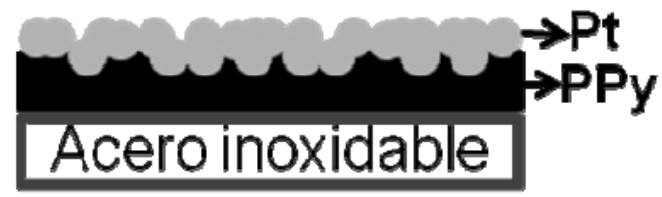

a)

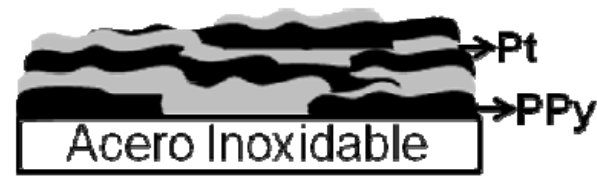

b)

Figura 1. Representación esquemática de la obtención de PPy/Pt a) bicapa y b) multicapa.

Los experimentos fueron clasificados en dos grupos: bicapa y multicapa, como se observa en la Tabla 1 y Fig. 1. En el primer experimento, se obtuvo una película de PPy en modo galvanostático, aplicando 0,001 A durante un tiempo de $2400 \mathrm{~s}$, en una disolución de Pirrol 0,100 M, en presencia del electrólito $\left(\mathrm{H}_{2} \mathrm{SO}_{4} \mathrm{0,100}\right.$ M). En la misma disolución se obtuvieron depósitos de películas de PPy en modo potenciostático, aplicando un potencial de $0,5 \mathrm{~V}$ durante un tiempo de $300 \mathrm{~s}$. Para obtener películas de PPy/Pt bicapa, sobre las películas de PPy obtenidas galvanostáticamente, se electrodepositó catódicamente platino a partir de la sal ( $\left[\left(\mathrm{NH}_{4}\right)_{2} \mathrm{PtCl}_{6}\right]$ 0,001 $\mathrm{M}+\mathrm{H}_{2} \mathrm{SO}_{4}$ 0,501 M), en modo galvanostático PPy/Pt 1 y potenciostático $\mathrm{PPy} / \mathrm{Pt}$ 2. En el segundo experimento se electrodepositaron películas multicapa de PPy/Pt 3 modo galvanostático y multicapa de PPy/Pt 4 modo potenciostático. Para ambos modos de electrodeposición, la disolución del electrólito fue (Pirrol 0,100 M + ([( $\left.\left(\mathrm{NH}_{4}\right)_{2} \mathrm{PtCl}_{6}\right]$ 0,001 M + $\mathrm{H}_{2} \mathrm{SO}_{4}$ 0,501 M). Las corrientes aplicadas en modo galvanostático fueron: primero de 0,001 A durante 200 s, para depositar el PPy y luego de $-0,005$ A por un tiempo de 200 s, para depositar el Pt. Estos ciclos se repitieron durante un tiempo total de 
electrodeposición de 15-20 min, para finalmente obtener las películas multicapa PPy/Pt 3. Utilizando el modo potenciostático, se aplicó, alternadamente, un potencial anódico de $0,5 \mathrm{~V}$ para oxidar el monómero de Pirrol y formar el polímero y uno catódico de $-0,5 \mathrm{~V}$ para reducir el platino. Cada potencial se aplicó durante un tiempo de $30 \mathrm{~s}$. Así se consiguieron las películas multicapa $\mathrm{PPy} / \mathrm{Pt} 4$.

\section{Resultados y discusión}

\section{Electropolimerización de pirrol}

En la

Figura 2a se presenta el comportamiento del potencial cuando se ha aplicado una corriente de 0,001 A. Se observa que el tiempo para alcanzar el potencial de oxidación del pirrol es de $450 \mathrm{~s}$, aproximadamente. Este tiempo es empleado en la pasivación del sustrato, una vez se alcanza un potencial de $0,5 \mathrm{~V}$, correspondiente al potencial de oxidación del monómero de pirrol, el potencial permanece constante dando lugar a la formación del polímero. La

Figura 2b ilustra la electrodeposición de PPy aplicando un potencial anódico de

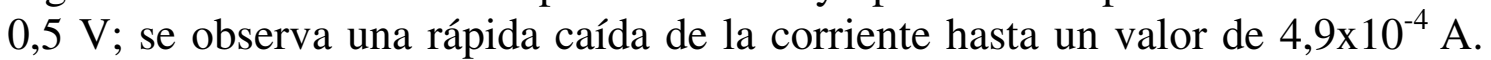
Esta caída es debida a la pasivación del sustrato. Las películas de PPy presentaron un espesor aproximado de $1,28 \mu \mathrm{m}$.

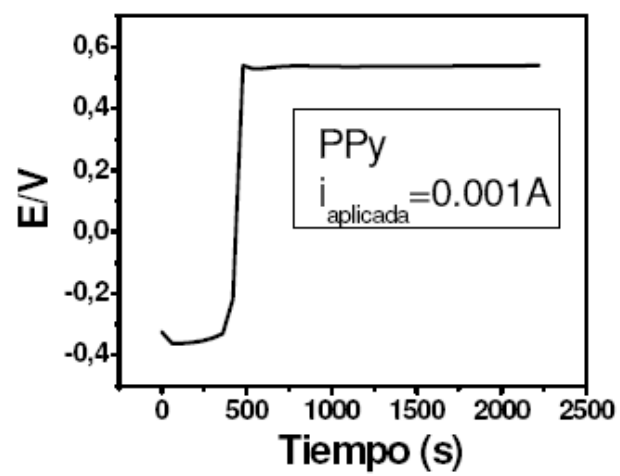

(a)

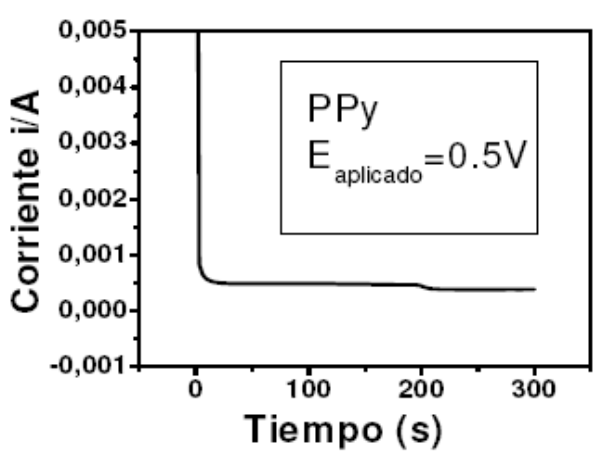

(b)

Figura 2. Electrodeposición de PPy: a) modo galvanostático; b) modo potenciostático.

En la Figura 3 se observan las fotografías de las películas de polipirrol obtenidas por ambos modos, donde puede notarse la diferencia de nucleación en la formación del polímero sobre el sustrato. La película formada en modo potenciostático, resultó ser más porosa y heterogénea que la obtenida galavanostáticamente. Esto es debido, posiblemente, a que en modo galavanostático se tiene un mayor control de la carga y del proceso de electrodeposición, dado que primero se da la pasivación del sustrato y luego la electropolimerización. Estas diferencias se ven reflejadas, igualmente, en la morfología de cada película, como se evidenciará posteriormente en este trabajo mediante análisis de microscopía electrónica de barrido. 


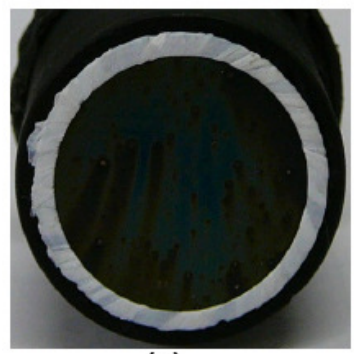

(a)

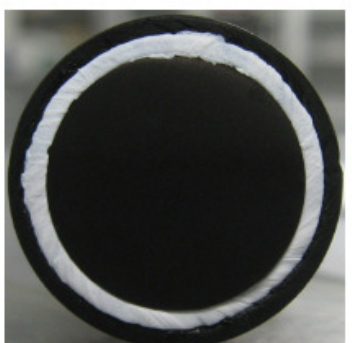

(b)

Figura 3. Fotografías de PPy obtenido por electrodeposición: a) pontenciostática y b) galvanostática.

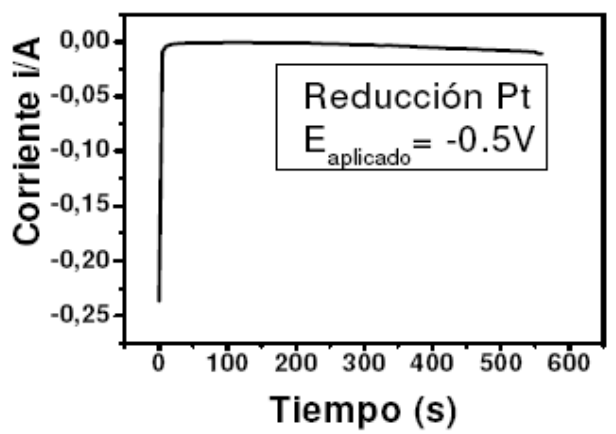

(a)

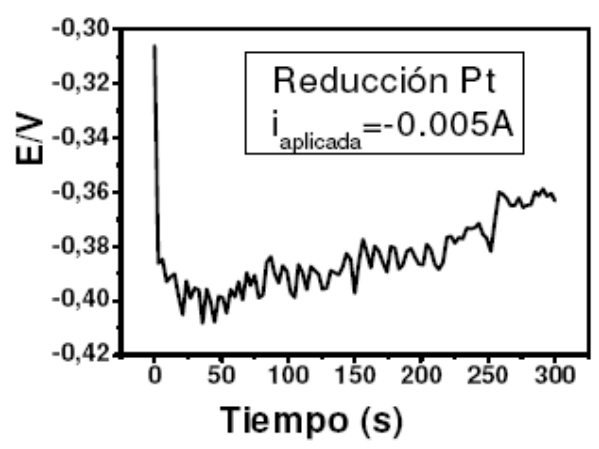

(b)

Figura 4. Reducción catódica de Pt por modo a) potenciostático, b) galvanostático.

\section{Electrodeposición de platino por reducción catódica y formación de películas PPy/Pt bicapa}

Sobre la película de PPy formada en modo galvanostático se realizaron, seguidamente, reducciones de $\mathrm{Pt}$ (IV) a $\mathrm{Pt}^{0}$ en modo potenciostático, aplicando un potencial de $-0,5 \mathrm{~V}$ y en modo galvanostático, aplicando una corriente de $-0,005$ A, en experimentos independientes. En la Figura 4a se presenta la reducción de platino en modo potenciostático. La respuesta en corriente de -0,001 A permaneció constante en el tiempo, permitiendo la deposición del platino sobre polipirrol PPy/Pt bicapa 1. En la Figura 4b, PPy/Pt bicapa 2, se presenta la reducción de platino sobre el PPy de modo galvanostático. En este caso, el potencial no alcanzó un valor estable, debido a la evolución de $\mathrm{H}_{2}$ sobre el electrodo de trabajo; por tanto, hay que presumir que el platino no alcanzó a formar un depósito homogéneo. A partir de la Figura 4a se cálculo el contenido de platino en las películas $\mathrm{PPy} / \mathrm{Pt}$ bicapa 1 usando la carga faradaica, obteniéndose que hay $0,14 \mathrm{mg} / \mathrm{cm}^{2}$, que corresponde a un $39,62 \%$ en peso de $\mathrm{Pt}$, en toda la película. Las películas bicapa presentaron un espesor aproximado de $1,42 \mu \mathrm{m}$.

\section{Obtención de películas PPy/Pt multicapa}

Las electrodeposiciones en las que se consiguieron depósitos de las películas $\mathrm{PPy} / \mathrm{Pt}$ multicapa 3 modo galvanostático y PPy/Pt multicapa 4 modo potenciostático, se ilustran en la Figura 5. En la Figura 5a se presenta la respuesta 
al potencial de la electrodeposión galvanostática de las películas $\mathrm{PPy} / \mathrm{Pt}$ multicapa 3. En la Figura 5 b se presenta la respuesta, en corriente, de la electrodeposición potenciostática de las películas multicapa PPy/Pt 4. Estas películas presentaron un espesor aproximado de 1,98 $\mu \mathrm{m}$. En ambos sistemas se evidencian ciclos escalonados de potencial o corriente constantes con respecto al tiempo, indicando la formación uniforme de los recubrimientos. Con la Figura 5b se obtienen los datos del contenido de platino en las películas PPy/Pt 4 usando la carga faradaica, donde hay $0,09 \mathrm{mg} / \mathrm{cm}^{2}$, lo que corresponde a un $25 \%$ en peso de Pt en cada película.

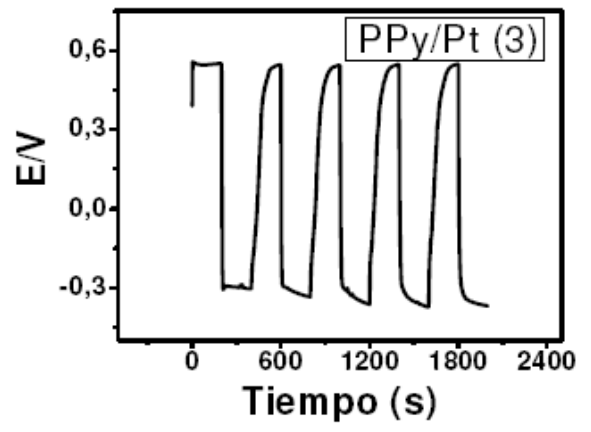

(a)

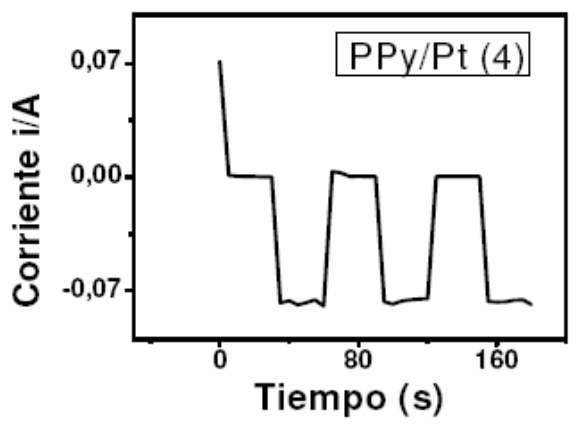

(b)

Figura 5. Obtención de multicapas PPy/Pt: a) PPy/Pt 3; b) PPy/Pt 4.

\section{Análisis Raman}

En las Figura 6a y 6b se presentan los espectros Raman correspondientes a las películas de PPy y PPy/Pt bicapa 1. En ambos espectros se pueden identificar las bandas características del polipirrol: 1592, 1369, 1260, 1060 y $932 \mathrm{~cm}^{-1}$ [33]. Las bandas que aparecen a 1592 y $1595 \mathrm{~cm}^{-1}$ corresponden al estiramiento $\mathrm{C}=\mathrm{C}$ a lo largo de la cadena del polímero [6,10,34]. Las bandas correspondientes a $1060 \mathrm{y}$ $930 \mathrm{~cm}^{-1}$ representan la deformación en el plano del enlace C-H del PPy en su forma oxidada. Debido a la intensidad de estas bandas y de acuerdo a lo reportado en la literatura, puede concluirse que el polímero presenta una alta conductividad $[6,10,35]$. La única diferencia notable entre ambos espectros, es la intensidad de las bandas; en el caso de la película de PPy/Pt bicapa 1 la intensidad es mayor que la de la película de PPy.

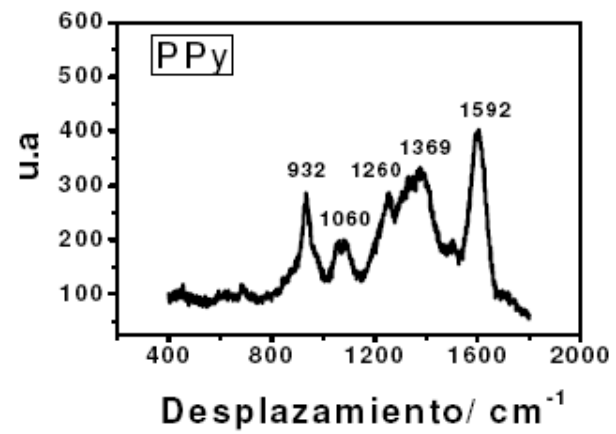

(a)

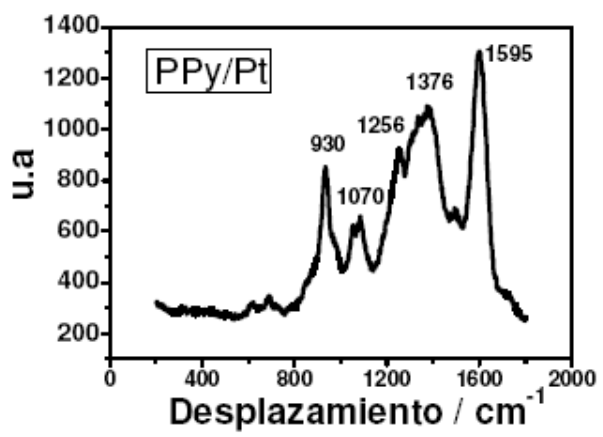

(b)

Figura 6. Espectros Raman de las películas de: a) PPy; b) PPy/Pt. 
La intensidad de las bandas en los espectros Raman para polímeros conductores, se ha asociado con la conductividad [6,10]. Por lo tanto, como es de esperar, las películas de $\mathrm{PPy} / \mathrm{Pt}$ presentan mayores valores de conductividad que las de polipirrol solo.
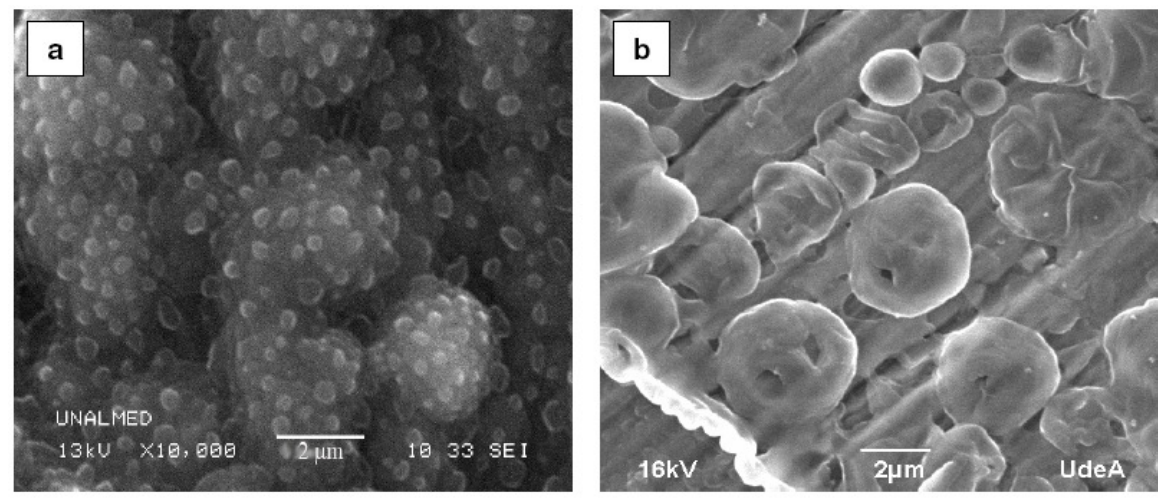

Figura 7. Micrografías MEB de películas de PPy formadas por modo: a) galvanostático, b) potenciostático.

\section{Análisis morfológico}

En la Figura 7 se presentan las micrografías (MEB) de las películas de PPy obtenidas en modo potenciostático y galvanostático. La micrografía de la Figura 7a corresponde al PPy obtenido galvanostáticamente. Puede apreciarse que se ha formado sobre el acero inoxidable siguiendo las líneas del pulido del sustrato,

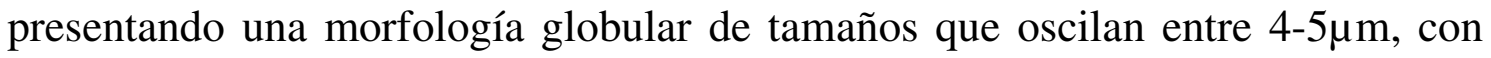
algunos núcleos exteriores más pequeños sobre la superficie de los glóbulos grandes, los cuales se resaltan sobre toda la matriz del polímero. Esta morfología globular, según trabajos anteriores, es típica del polipirrol cuando se forma por electropolimerización [36-38]. En la micrografía de la Figura 7b se puede apreciar una distribución heterogénea del PPy sobre la superficie del sustrato, dado que no se presenta una geometría definida. Se puede apreciar que las morfologías de ambas películas son diferentes. Además, se observa que la morfología del PPy viene afectada por la técnica de electrodeposición elegida para su polimerización. Estos resultados están de acuerdo con las conclusiones obtenidas anteriormente acerca de las ventajas de tener más control de la carga sobre la formación del depósito.

En la Figura 8 se presentan las micrografías para las películas PPy/Pt bicapa y multicapa. La morfología observada en la Figura 8a corresponde a películas de PPy/Pt bicapa 1. Se puede apreciar una morfología globular que sigue las líneas del pulido del sustrato de acero. Dada la morfología observada en la Figura 7a para el PPy, puede concluirse que el platino en la Figura 8a esta recubriendo el polímero, adoptando su morfología y que cubre homogéneamente su superficie, ya que no se observan los núcleos exteriores como ocurre en la película de PPy. En la Figura 8b se observa la morfología correspondiente a películas de $\mathrm{PPy} / \mathrm{Pt}$ multicapa 4, en donde puede notarse la formación globular sobre la superficie del sustrato y cómo el platino deja aleatoriamente algunas zonas del PPy 
descubiertas, como resultado de alternar los potenciales de deposición en tiempos tan cortos, presentándose la formación discontinua de regiones cubiertas por el polímero (regiones negras) y por el platino (regiones grises). Para ambos sistemas de $\mathrm{PPy} / \mathrm{Pt}$, se evidencia que el platino cubre la superficie del PPy adoptando su morfología.
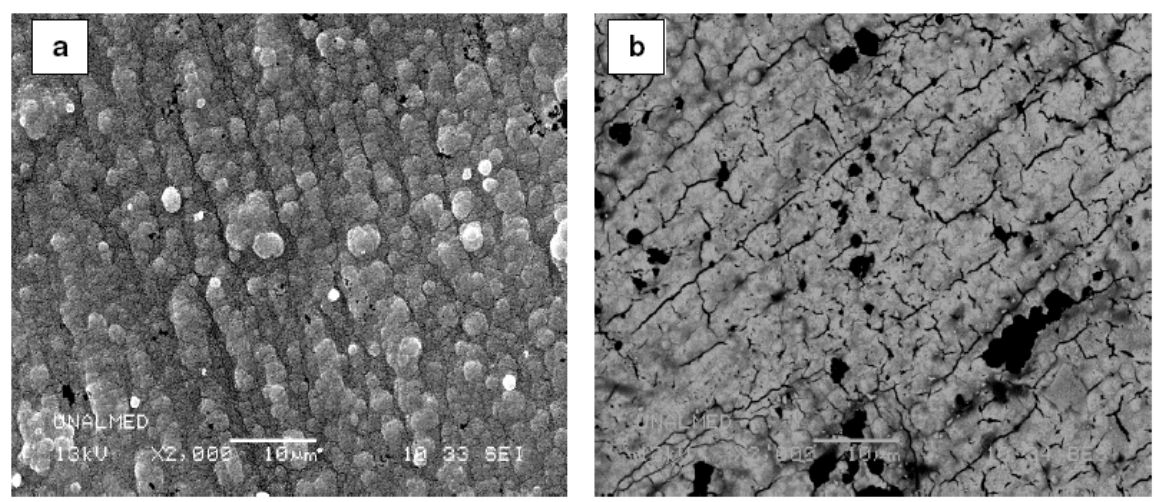

Figura 8. Micrografías MEB de películas: a) PPy/Pt bicapa 1 y b) PPy/Pt multicapa 4.

\section{Medidas de conductividad eléctrica (AC)}

En la Tabla 2 se presentan los resultados de conductividad obtenidos para PPy, $\mathrm{PPy} / \mathrm{Pt}$ bicapa 1 y PPy/Pt multicapa 4. Estos valores fueron obtenidos a partir de medidas de impedancia en estado sólido $[39,40]$. Se puede apreciar como la conductividad del polímero aumenta con la cantidad de platino depositado en las películas, como era de esperarse. Sin embargo, se observa como en la película PPy/Pt multicapa 4 el valor de conductividad no aumenta significativamente, dado el porcentaje en peso de platino que contiene la película, calculado a partir de la carga faradáica en la Figura 5b. Por el contrario, en la película $\mathrm{PPy} / \mathrm{Pt}$ bicapa 1 se evidencia un aumento significativo, lo que indica que estas películas pueden presentar una actividad catalítica mayor ya que este depende directamente del contenido de platino.

Tabla 2. Resultados de conductividad para las películas de PPy, PPy/Pt bicapa 1, PPy/Pt multicapa 4.

\begin{tabular}{||c|c||}
\hline \hline Muestra & Conductividad $\left(\mathbf{S} \cdot \mathbf{c m}^{-\mathbf{1}}\right)$ \\
\hline PPy & $1,41 \times 10^{-4}$ \\
\hline PPy/Pt bicapa $\mathbf{1}$ & $1,00 \times 10^{-2}$ \\
\hline PPy/Pt multicapa $\mathbf{4}$ & $1,39 \times 10^{-4}$ \\
\hline
\end{tabular}

\section{Voltametría cíclica}

Con el fin de observar el potencial de oxidación del polipirrol y su variación debida a la incorporación de platino, se realizaron curvas de voltametría cíclica. En la Figura 9 se muestran las curvas de voltametría cíclica para las películas de acero 304, PPy y PPy/Pt bicapa y multicapa, usando como electrólito una 
disolución de $\mathrm{H}_{2} \mathrm{SO}_{4}$ 0,501 M. La Figura 9a ilustra las voltametrías realizadas al sustrato de acero inoxidable y a la película de PPy en dicho medio. El sustrato de acero inoxidable 304 presenta dos potenciales de oxidación, el primero a un potencial de $-0,3 \mathrm{~V}$ atribuido a la pasivación y un segundo a un potencial de 1,4 $\mathrm{V}$ correspondiente a la oxidación del sustrato en este medio. En la curva correspondiente al PPy puede observarse la sobreoxidación del polímero a 1,0 V y un potencial de reactivación en el sentido anódico del voltamograma a 1,4 V que, como se vio para el sustrato desnudo, este pico corresponde a la oxidación del acero. El pico a 1,4 V en la curva de retorno para el PPy indica, muy posiblemente, el desprendimiento de la película después de pasar la sobre oxidación. En la Figura 9 b se presentan las voltametrías realizadas a las películas PPy/Pt bicapa 1 y PPy/Pt multicapa 4 en 0,501 $\mathrm{M} \mathrm{H}_{2} \mathrm{SO}_{4}$. La película $\mathrm{PPy} / \mathrm{Pt}$ bicapa 1 presentan un pico de oxidación, en $0,97 \mathrm{~V}$ y un pico de reactivación en 1,4 V. El primer potencial corresponde a la sobre-oxidación del polipirrol, igual que el observado en la Figura 9a [8,41]. El segundo pico es atribuido a la oxidación del sustrato, como se dijo anteriormente. En la curva obtenida para PPy/Pt multicapa 4 se observa una insinuación de pico para la sobre-oxidación del PPy a 1,0 V; posteriormente, se evidencia que la película nuevamente se desprende del sustrato, dado el pico a 1,4 V, el cual es atribuido a la oxidación del sustrato desnudo. Comparando con el voltamograma obtenido para el polímero, puede concluirse que el platino no inhibe la oxidación de éste en ninguno de los casos. Se observa un ligera diferencia en las corrientes de oxidación del polímero a 1,0 V, siendo mayor en la película bicapa que en la multicapa.

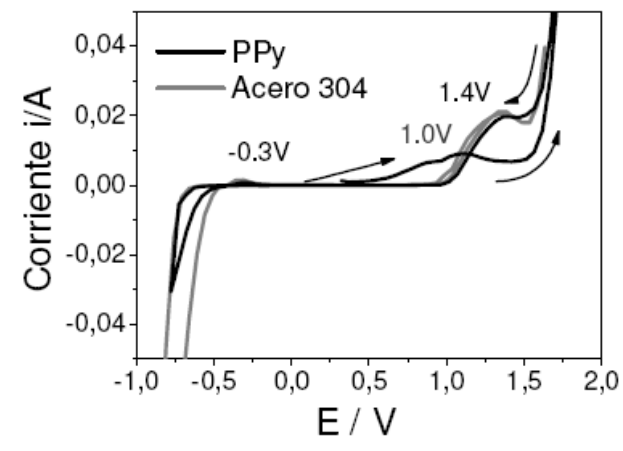

(a)

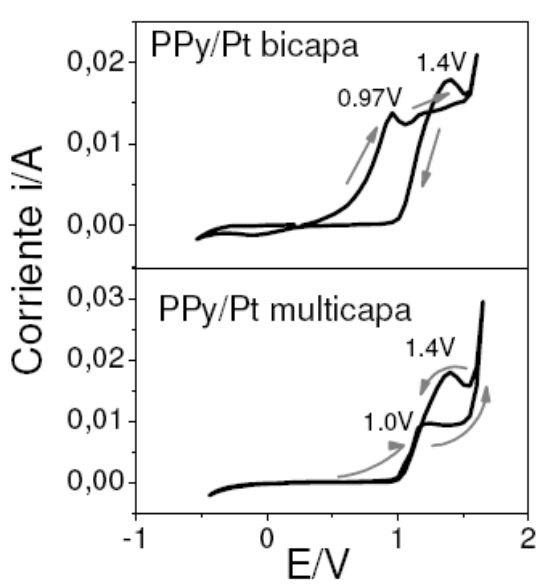

(b)

Figura 9. Voltametría cíclica en $\mathrm{H}_{2} \mathrm{SO}_{4} 0,5 \mathrm{M}$ para: a) acero y PPy; b) PPy/Pt 1 y $\mathrm{PPy} / \mathrm{Pt} 4$.

\section{Conclusiones}

Las películas de PPy obtenidas por electropolimerización de pirrol en modo galvanostático, resultaron menos porosas y más homogéneas que las obtenidas por modo potenciostático. De acuerdo con los espectros Raman, el platino no 
tuvo influencia en las modificaciones estructurales del polímero. Las imágenes SEM ilustran que la morfología de la película de PPy puro es granular, confirmándose lo reportado en la literatura. En las películas bicapa, el Pt recubre uniformemente la superficie, mientras que en las multicapa, el recubrimiento de Pt es parcial. Los valores de conductividad calculados a partir de los diagramas de impedancia, evidencian la incorporación del platino a la matriz polimérica de polipirrol, debido a que en las películas con platino se aumenta notablemente el valor de conductividad. De las curvas de voltametría cíclica puede interpretarse que el platino no inhibe la oxidación de polímero para ninguno de los sistemas PPy/Pt. Además, se observa una ligera diferencia en las corrientes de oxidación del polímero a 1,0 V, siendo mayor en la película $\mathrm{PPy} / \mathrm{Pt}$ bicapa que en la $\mathrm{PPy} / \mathrm{Pt}$ multicapa. Estos resultados se usaran como base para evaluar posteriormente dichas películas como superficies catalíticas para la oxidación de metanol y etanol, buscando su aplicación en celdas de combustible.

\section{Electrodeposición de Películas de Polipirrol/Platino}

\section{Resumen}

Las celdas de combustible son novedosas alternativas para la producción de energía con mínimo impacto ambiental. Los polímeros conductores se perfilan entre los materiales más prometedores para la fabricación de dichas celdas, en términos de su aplicación y operación, siendo el polipirrol (PPy) uno de ellos. En este trabajo se presenta la obtención de películas de PPy y PPy/Platino en diferentes configuraciones (bicapa y multicapa). Las películas se sintetizaron electroquímicamente sobre acero inoxidable 304, en presencia de monómero de pirrol y de la sal de hexacloroplatinato de amonio obtenida por tratamiento hidrometalúrgico. Se estudiaron la composición, la morfología, la conductividad y las propiedades electroquímicas de las películas, por espectroscopia Raman, microscopía electrónica de barrido, perfilometría, impedancia y se evaluaron sus potenciales de oxidación por voltametría cíclica, para evaluar su posible uso como electrodos para celdas de combustible.

Palabras Clave: Polipirrol/Platino, Polímeros conductores, Voltametría Cíclica.

\section{Referencias}

1. K. Bouzek, K.M. Mangold, K. Jüttner, J. Appl. Electrochem. 31 (2001) 501.

2. Y. Wang, D.O. Northwood, Thin Solid Films 516 (2008) 7427.

3. $\quad$ R. Ansari, E-J. Chem. 3 (2006) 186.

4. K. Bouzek, K.M. Mangold, K. Jüttner, Electrochim. Acta 46 (2001) 661.

5. K. Jüttner, K.M. Mangold, M. Lange, K. Bouzek, Russ. J. Electrochem. 40 (2004) 317.

6. W. Chen, C.M. Li, P. Chen, C.Q. Sun, Electrochim. Acta 52 (2007) 2845.

7. P. Holzhauser, K. Bouzek, Z. Bastl, Synth. Met. 155 (2005) 501.

8. S. Radhakrishnan, A. Adhikari, J. Power Sources 155 (2006) 157.

9. M.A.D. Valle, F.R. Díaz, M.E. Bodini, T. Pizarro, R. Córdova, H. Gómez, R. Schrebler, J. Appl. Electrochem. 28 (1998) 943.

10. M. Bazzaoui, J.I. Martins, E. Machnikova, E.A. Bazzaoui, L. Martins, Eur. Polym. J. 43 (2007) 1347. 
11. M.A.L. García, M.A. Smit, J. Power Sources 158 (2006) 397.

12. P. Herrasti, P. Ocón, Appl. Surf. Sci. 172 (2001) 276.

13. E. Hür, G. Bereket, Y. Sahin, Mater. Chem. Phys. 100 (2006) 19.

14. J.O. Iroh, K. Levine, Eur. Polym. J. 38 (2002) 1547.

15. S. Joseph, J.C. McClure, R. Chianelli, P. Pich, P.J. Sebastian, Int. J. Hydrog. Energy 30 (2005) 1339.

16. M.A. Malik, R. Wlodarczyk, P.J. Kulesza, H. Bala, K. Miecznikowski, Corros. Sci. 47 (2005) 771.

17. T.N. Murakami, M. Grätzel, Inorg. Chim. Acta 361 (2008) 572.

18. S.S. Pandey, W. Takashima, K. Kaneto, Thin Solid Films 438-439 (2003) 206.

19. W. Prissanaroon, N. Brack, P.J. Pigram, J. Liesegang, Curr. Appl. Phys. 4 (2004) 163.

20. R.M.G. Rajapakse, R.M.M.Y. Rajapakse, H.M.N. Bandara, B.S.B. Karunarathne, Electrochim. Acta 53 (2008) 2946.

21. Y.J. Ren, C.L. Zeng, J. Power Sources 182 (2008) 524.

22. C.K. Tan, D.J. Blackwood, Corros. Sci. 45 (2003) 545.

23. J. Tietje-Girault, C. Ponce de León, F.C. Walsh, Surf. Coat. Tech. 201 (2007) 6025.

24. T. Tüken, Surf. Coat. Tech. 200 (2006) 4713.

25. T. Tüken, B. Yazici, M. Erbil, Surf. Coat. Tech. 202 (2007) 425.

26. J. Wang, J. Chen, C.Y. Wang, D. Zhou, C.O. Too, G.G. Wallace, Synth. Met. 153 (2005) 117.

27. Y. Wang, D.O. Northwood, J. Power Sources 163 (2006) 500.

28. Y. Wang, D.O. Northwood, J. Power Sources 175 (2008) 40.

29. M.A. Malik, M.T. Galkowski, H. Bala, B. Grzybowska, P.J. Kulesza, Electrochim. Acta 44 (1999) 2157.

30. M. Trueba, S.P. Trasatti, S. Trasatti, Mater. Chem. Phys. 98 (2006) 165.

31. M. Georgieva, B. Andonovski, Anal. Bioanal. Chem. 375 (2003) 836.

32. J. Whalen III, J. Weiland, P. Searson, J. Electrochem. Soc. 152 (2005) C738.

33. M.J.L. Santos, A.G. Brolo, E.M. Girotto, Electrochim. Acta 52 (2007) 6141.

34. Y.C. Liu, J. Phys. Chem. B 108 (2004) 2948.

35. H. Nguyen Thi Le, M.C. Bernard, B. Garcia-Renaud, C. Deslouis, Synth. Met. 140 (2004) 287.

36. I.L. Lehr, S.B. Saidman, Corrosion Science 49 (2007) 2210.

37. X. Lu, D. Chao, J. Chen, W. Zhang, Y. Wei, Mater. Lett. 60 (2006) 2851.

38. N.C.T. Martins, T. Moura e Silva, M.F. Montemor, J.C.S. Fernandes, M.G.S. Ferreira, Electrochim. Acta 53 (2008) 4754.

39. R. Suvarna, K. Rao, K. Subbarangaiah, Bull. Mat. Sci. 25 (2002) 647.

40. T. Vishnuvardhan, V. Kulkarni, C. Basavaraja, S. Raghavendra, Bull. Mat. Sci. 29 (2006) 77.

41. S. Lamprakopoulos, D. Yfantis, A. Yfantis, D. Schmeisser, J. Anastassopoulou, T. Theophanides, Synth. Met. 144 (2004) 229. 